\title{
Renal cell carcinoma associated with tuberous sclerosis complex (TSC)/mammalian target of rapamycin (MTOR) genetic alterations
}

(c) The Author(s), under exclusive licence to United States \& Canadian Academy of Pathology 2022

Modern Pathology (2022) 35:296-297; https://doi.org/10.1038/ s41379-021-00971-y

The majority of hereditary tumor syndromes involve germline mutations, which effectively inactivate tumor suppressor genes (for example TSC1 and TSC2), whereby cells with a bi-allelic inactivation of such genes originate tumors in a classic tumor suppressor "two-hit" paradigm. The diverse morphologic spectrum of neoplasms found in the tuberous sclerosis complex (TSC) integrates many fundamentals of cellular biology, with the MTOR pathway showing frequent activation, as evidenced by expression of multiple downstream targets of MTOR and an increased neoplastic cell size (now well known to be a functional consequence of enhanced MTOR activity) ${ }^{1}$.

While angiomyolipomas and cysts are the most common renal manifestations of TSC, $\sim 4 \%$ of patients will develop renal cell carcinoma $(\mathrm{RCC})^{2}$. Careful morphologic studies of these RCC have permitted their subdivision into three main groups: first, RCC with clear cytoplasm, papillary architecture, and prominent smooth muscle stroma; second, RCC with granular eosinophilic cytoplasm and macrocystic architecture; and third, RCC resembling the eosinophilic variant of chromophobe $\mathrm{RCC}^{3,4}$. One would predict that there should be sporadic counterparts to each of these neoplasms, effected by somatic mutations in the TSC1/2 genes in patients who do not suffer from the tuberous sclerosis complex. Identification of such neoplasms, however, has been confounded by the fact that the $T S C 1 / 2$ genes, as well as the gene coding the downstream protein mammalian target of rapamycin (MTOR), are mutated secondarily in $\sim 5 \%$ of the more common subtypes of RCC, including bonafide clear cell and chromophobe RCC cases ${ }^{5,6}$. Parenthetically, these mutations may be associated with clinical response to MTOR inhibitors, emphasizing their therapeutic relevance. However, in recent years and in several studies in the current issue of Modern Pathology, sporadic counterparts to the hereditary tuberous sclerosis complexassociated RCC that are associated with somatic TSC/MTOR pathway mutations have now been described.

First, representing a somatic counterpart to group one, a RCC characterized by prominent fibromuscular brands transecting acinar and tubulopapillary architecture, and clear cells with voluminous cytoplasm and well-defined cell borders ${ }^{7}$ has been demonstrated to harbor somatic TSC or MTOR mutations ${ }^{8,9}$. Interestingly, these neoplasms demonstrate morphologic overlap with renal tumors recently described as harboring mutations in the ELOC (formerly known as TCEB1) gene at 8q21.11, in an absence of concurrent $V H L$ or chromosome $3 p$ alterations ${ }^{10}$. These neoplasms typically label for cytokeratin 7 and carbonic anhydrase IX like clear cell papillary RCC/renal angiomyoadenomatous tumor, but in contrast also label for CD10, have voluminous cytoplasm, and lack subnuclear vacuolization. Second, corresponding to group 2, eosinophilic solid and cystic RCC (ESC RCC), characterized by solid and cystic architecture, polygonal neoplastic cells with voluminous eosinophilic cytoplasm and basophilic cytoplasmic stippling, and frequent patchy immunoreactivity for cytokeratin $20^{11}$, has been shown to harbor somatic TSC1 or TSC2 mutations ${ }^{12-14}$. While typically indolent and presenting in adult females, this neoplasm often occurs in children ${ }^{15}$ and may rarely present with metastatic disease which in one case has dramatically responded to treatment with MTOR inhibitors. Finally, corresponding to group three, a neoplasm provisionally termed "low-grade oncocytic tumor" (LOT) ${ }^{16}$ has been described and in this issue of Modern Pathology demonstrated to be associated with somatic TSC1/2 gene mutations ${ }^{17-19}$. These neoplasms resemble the eosinophilic variant of chromophobe RCC in that they are composed of solid areas of low-grade oncocytic cells with perinuclear halos, which label with cytokeratin 7 . In contrast, however, the neoplasm typically features transitions from solid growth to elongated cords/sheaths of neoplastic cells in edematous stroma, has fewer nuclear irregularities and less welldeveloped perinuclear halos, and does not label diffusely for CD117. Not surprisingly, LOT shares morphologic and biomarker overlap with a subset of eosinophilic variant of chromophobe RCC described in literature with negative CD117 expression and presence of MTOR mutations ${ }^{20}$. Apart from the sporadic and hereditary settings, LOT have now also been reported in patients with end-stage renal disease (ESRD) ${ }^{21}$.

Also included among chromophobe RCC-like tumors in patients with tuberous sclerosis complex are neoplasms with hyalinized stroma, prominent nucleoli, and eosinophilic cytoplasm with prominent cytoplasmic vacuoles ${ }^{22}$. Such tumors were originally described in nonsyndromic patients on morphologic grounds under the term "high-grade oncocytic tumor"23, given their prominent nucleoli; however, these neoplasms typically lack mitoses and have thus far have had benign outcomes. Somatic TSC or MTOR mutations ${ }^{24}$ have now been found in these neoplasms (reported under the term eosinophilic and vacuolated RCC). These neoplasms are recognized as an evolving entity by both the International Society of Urologic Pathology ${ }^{25}$, and Genitourinary Pathology Society ${ }^{26}$, and the latter has proposed the provisional term eosinophilic and vacuolated tumor (EVT). An additional study reported in this issue of Modern Pathology confirms the presence of somatic TSC/MTOR mutations in $\mathrm{EVT}^{27}$.

While remarkable progress has been made over the past decade in the delineation of TSC/MTOR-mutation-related renal neoplasia in both of the hereditary and sporadic settings ${ }^{28}$, several questions remain to be answered. One query is whether there are other distinctive RCCs that may prove to be associated with TSC1/ 2 or MTOR gene mutations. Along these lines, a recent report 
described TSC2 mutations in a subset of acquired cystic diseaseassociated $\mathrm{RCC}^{29}$. Another is how frequent are TSC1/2 or MTORmutated RCC that do not cleanly fit a given category but instead have overlapping features of ESC, LOT, and/or EVT. In addition, it is currently not very clear how TSC related neoplasia may be affected at the phenotypic and molecular level by aberrations involving other genes from related/unrelated pathways; for example, deletions involving TSC2 and the adjacent PKD1 gene have been linked to a distinct subtype of TSC exhibiting significant cystic kidney disease ${ }^{30}$. Further studies in the upcoming few years should help further clarify this fascinating area of study with a successful definition of specific genomic insults that link aberrations in TSC genes with a spectrum of morphologic and clinical phenotypes.

Finally, the current National Comprehensive Cancer Network as well as American Urology Association guidelines recommend these clinical criteria for genetic risk evaluation of patients with potential hereditary kidney cancer: diagnosis at age equal to or less than 46 years, bilateral or multifocal tumors, and a first degree relative with RCC. However, specific pathologic findings (such as succinate dehydrogenase deficient or Birt-Hogg-Dubérelated tumor histology) should also trigger such evaluation. Indeed, pathologists not infrequently suggest the genetic consultation and germline testing that first establishes the diagnosis of hereditary renal neoplasia in a family following examination of a renal core biopsy or resection in a proband. The expanding spectrum of TSC-related renal neoplasia may further expand the pivotal role pathologists play in the initial identification of hereditary renal neoplasia.

Pedram Argani ${ }^{1 凶}$ and Rohit Mehra (iD ${ }^{2}$ 'Department of Pathology, Johns Hopkins Medical Institutions, Baltimore, MD, USA. ${ }^{2}$ Department of Pathology, University of Michigan, Ann Arbor, MI, USA. ${ }^{\boxplus e m a i l: ~ p a r g a n i @ j h m i . e d u ~}$

\section{REFERENCES}

1. Fingar, D. C., Salama, S., Tsou, C., Harlow, E. \& Blenis, J. Mammalian cell size is controlled by mTOR and its downstream targets S6K1 and 4EBP1/elF4E. Genes Dev. 16, 1472-1487 (2002).

2. Rakowski, S. K. et al. Kidney Int. 70, 1777-1782 (2006).

3. Guo, J. et al. Tuberous sclerosis-associated renal cell carcinoma. A clinicopathologic study of 57 separate carcinomas in 18 patients. Am. J. Surg. Pathol. 38, 1457-1467 (2014).

4. Yang, P. et al. Renal cell carcinoma in tuberous sclerosis complex. Am. J. Surg. Pathol. 38, 895-909 (2014).

5. Maroto, P. et al. Biallelic TSC2 mutations in a patient with chromophobe renal cell carcinoma showing extraordinary response to temsirolimus. J. Natl. Compr. Cancer Netw. 16, 352-358 (2018).

6. Kwiatkowski, D. J. et al. Mutations in TSC1, TSC2, and MTOR are associated with response to rapalogs in pateints with metastatic renal cell carcinoma. Clin. Cancer Res. 22, 2445-2452 (2016).

7. Williamson, S. R. et al. Renal cell carcinoma with angioleiomyoma-like stroma: clinicopathologic, immunohistochemical, and molecular features supporting classification as a distinct entity. Mod. Pathol. 28, 279-294 (2015).

8. Shah, R. B. et al. "Renal cell carcinoma with fibroleiomyomatous stroma" harbor somatic mutations of TSC1, TSC2, MTOR, and/or ELOC (TCEB1): Clinicopathologic and molecular characterization of 18 sporadic tumors. Supports a distinct entity. Am. J. Surg. Pathol. 44, 571-581 (2020).

9. Parilla, M. et al. Genetic underpinnings of renal cell carcinoma with leiomyomatous stroma. Am. J. Surg. Pathol. 43, 1135-1144 (2019).

10. Hakimi, A. A. et al. TCEB1-mutated renal cell carcinoma: a distinct genomic and morphological subtype. Mod. Pathol. 28, 845-853 (2015).
11. Trpkov, K. et al. Eosinophilic, solid, and cystic renal cell carcinoma. Clinicopathologic study of 16 unique, sporadic neoplasms occurring in women. Am. J. Surg. Pathol. 40, 60-71 (2016).

12. Palsgrove, D. N. et al. Eosinophilic solid and cystic (ESC) renal cell carcinomas harbor TSC mutations: molecular analysis supports an expanding clinicopathologic spectrum. Am. J. Surg. Pathol. 42, 1166-1181 (2018).

13. Mehra, R. et al. Somatic Bi-allelic loss of TSC genes in eosinophilic solid and cystic renal cell carcinoma. Eur. Urol. 74, 483-486 (2018).

14. Parilla, M. et al. Are sporadic eosinophilic solid and cystic renal cell carcinomas characterized by somatic tuberous sclerosis gene mutations. Am. J. Surg. Pathol. 42, 911-917 (2018)

15. Li, Y. et al. Re-evaluation of 33 'unclassified' eosinophilic renal cell carcinomas in young patients. Histopathology 72, 588-600 (2018).

16. Trpkov, K. et al. Low-grade oncocytic tumour of kidney (CD117-negative, cytokeratin 7 positive): a distinct entity? Histopathology 75, 174-184 (2019).

17. Kapur, P. et al. Germline or sporadic mTOR pathway mutations in low-grade oncocytic tumor of the kidney. Mod. Pathol. https://doi.org/10.1038/s41379-02100896-6 (2021).

18. Morini, A. et al., Low-grade oncocytic renal tumor (LOT): mutations in mTOR pathway genes and low expression of FOXI1. Mod. Pathol. https://doi.org/ 10.1038/s41379-021-00906-7 (2021).

19. Mohanty S., Satapathy A., Aggarwal A. Oncocytic renal neoplasms with diffuse cytokeratin 7 immunohistochemistry harbor frequent alterations in the mammalian target of rapamycin pathway. Mod. Pathol. (2021). https://doi.org/10.1038/ s41379-021-00969-6. Online ahead of print.

20. Skala, S. L. et al. Next-generation RNA sequencing-based biomarker characterization of chromophobe renal cell carcinoma and related oncocytic neoplasms. Eur. Urol. 78, 63-74 (2020).

21. Kravtsov, O. et al. Low-Grade Oncocytic Tumor of Kidney (CK7-Positive, CD117Negative): Incidence in a single institutional experience with clinicopathological and molecular characteristics. Hum. Pathol. 114, 9-18 (2021).

22. Trpkov, K. et al. High-grade oncocytic tumour (HOT) of kidney in a patient with tuberous sclerosis complex. Histopathology 75, 440-445 (2019).

23. He, H. et al. "High-grade oncocytic renal tumor": morphologic, immunohistochemical, and molecular genetic study of 14 cases. Virchows Arch. 473, 725-738 (2018).

24. Chen, Y. B. et al. Somatic mutations of TSC2 or MTOR characterize a morphologically distinct subset of sporadic renal cell carcinoma with eosinophilic and vacuolated cytoplasm. Am. J. Surg. Pathol. 43, 121-131 (2019).

25. Williamson, S. R. et al. Report from the International Society of Urological Pathology (ISUP) consultation conference on molecular pathology of urogenital cancers. III: Molecular pathology of kidney cancer. Am. J. Surg. Pathol 44, e47-e65 (2020).

26. Trpkov, K. et al. New developments in existing WHO entities and evolving molecular concepts: The Genitourinary Pathology Society (GUPS) update on renal neoplasia. Mod. Pathol. 34, 1392-1424 (2021).

27. Farcas, M. et al. Eosinophilic vacuolated tumor (EVT) of kidney demonstrates sporadic TSC/MTOR mutations: next generation sequencing multi-institutional study of 19 cases. Mod. Pathol. https://doi.org/10.1038/s41379-021-00941-42021 (2021).

28. Tjota, M. et al. Eosinophilic renal cell tumors with a TSC and MTOR gene mutations are morphologically and immunohistochemically heterogenous. Clinicopathologic and molecular study. Am. J. Surg. Pathol. 44, 943-954 (2020).

29. Shah, A. et al. Acquired cystic kidney disease-associated renal cell carcinoma (ACKD-RCC) harbor recurrent mutations in KMT2C and TSC2 genes. Am. J. Surg. Pathol. 44, 1479-1486 (2020).

30. Sampson, J. R. et al. Renal cystic disease in tuberous sclerosis: role of the polycystic kidney disease 1 gene. Am. J. Hum. Genet. 61, 843-851 (1997).

\section{ADDITIONAL INFORMATION}

Correspondence and requests for materials should be addressed to Pedram Argani.

Reprints and permission information is available at http://www.nature.com/ reprints

Publisher's note Springer Nature remains neutral with regard to jurisdictional claims in published maps and institutional affiliations. 